\title{
Weak Galactic halo-Fornax dSph connection from RR Lyrae stars
}

\author{
G. Fiorentino ${ }^{1}$, M. Monelli ${ }^{2,3}$, P. B. Stetson ${ }^{4}$, G. Bono ${ }^{5,6}$, C. Gallart ${ }^{2,3}$, C. E. Martínez-Vázquez ${ }^{2,3}$, \\ E. J. Bernard ${ }^{7}$, D. Massari ${ }^{1,8}$, V. F. Braga ${ }^{5}$, and M. Dall'Ora ${ }^{9}$
}

\author{
1 INAF-Osservatorio Astronomico di Bologna, via Ranzani 1, 40127 Bologna, Italy \\ e-mail: giuliana.fiorentino@oabo.inaf.it \\ 2 IAC-Instituto de Astrofísica de Canarias, 38205 La Laguna, Tenerife, Spain \\ 3 ULL-Universidad de La Laguna, Dpto. Astrofísica, 38206 La Laguna, Tenerife, Spain \\ ${ }^{4}$ Herzberg Astronomy and Astrophysics, National Research Council Canada, 5071 West Saanich Road, Victoria, BC V9E 2E7, \\ Canada \\ 5 Dipartimento di Fisica, Università di Roma Tor Vergata, via della Ricerca Scientifca 1, 00133 Roma, Italy \\ 6 INAF-Osservatorio Astronomico di Roma, via Frascati 33, 00040 Monteporzio Catone, Italy \\ 7 Laboratoire Lagrange, Observatoire de la Cote d'Azur, 06304 Nice Cedex 4, France \\ 8 University of Groningen, Kapteyn Astronomical Institute, 9747 AD Groningen, The Netherlands \\ 9 INAF-Osservatorio Astronomico di Capodimonte, salita Moiariello 16, 80131 Napoli, Italy
}

Received 8 August 2016 / Accepted 9 December 2016

\begin{abstract}
Aims. For the first time accurate pulsation properties of the ancient variable stars of the Fornax dwarf spheroidal galaxy (dSph) are discussed in the broad context of galaxy formation and evolution.

Methods. Homogeneous multi-band BVI optical photometry of spanning twenty years has allowed us to identify and characterize more than 1400 RR Lyrae stars (RRLs) in this galaxy.

Results. Roughly $70 \%$ are new discoveries. We investigate the period-amplitude distribution and find that Fornax shows a lack of high amplitude $\left(A_{V} \gtrsim 0.75 \mathrm{mag}\right)$ short period fundamental-mode RRLs $(P \lesssim 0.48 \mathrm{~d}$, HASPs). These objects occur in stellar populations more metal-rich than $[\mathrm{Fe} / \mathrm{H}] \sim-1.5$ and they are common in the Galactic halo (hereafter Halo) and in globulars. This evidence suggests that old Fornax stars (older than $10 \mathrm{Gyr}$ ) are relatively metal poor. A detailed statistical analysis of the role of the present-day Fornax dSph in reproducing the Halo period distribution shows that it can only account for up to $20 \%$ of the Halo when combined with RRLs in massive dwarf galaxies (Sagittarius dSph, Large Magellanic Cloud). This finding indicates that Fornax-like systems played a smaller role than massive dwarfs in building up the Halo.

Conclusions. We also discuss the occurrence of HASPs in connection with the luminosity and the early chemical composition of nearby dwarf galaxies. We find that, independently of their individual star formation histories, bright $\left(M_{V} \lesssim-13.5\right.$ mag) galaxies have HASPs, whereas faint ones $\left(M_{V} \gtrsim-11 \mathrm{mag}\right)$ do not. Interestingly enough, Fornax belongs to a luminosity range $\left(-11<M_{V} \lesssim\right.$ $-13.5 \mathrm{mag}$ ) in which the occurrence of HASPs appears to be correlated with the early star formation and chemical enrichment of the host galaxy.
\end{abstract}

Key words. galaxies: evolution - galaxies: individual: Fornax dSph - stars: variables: RR Lyrae

\section{Introduction}

Our Local Group (LG) includes a sizable sample of dwarf galaxies located within the immediate surroundings of the two large spirals, the Milky Way (MW) and Andromeda (M31). Modern cosmological simulations predict that large galaxies assembled by means of merging processes starting from small protogalactic fragments (Searle \& Zinn 1978; Springel et al. 2006). Dwarf galaxies that we see today may be the relics of such fragments not cannibalized by the MW potential. Galactic archeology has been adopted to test this theoretical framework, with inconclusive results to date. In particular, the iron and the $\alpha$-element distributions (see Venn et al. 2004; Tolstoy et al. 2009; Feltzing \& Chiba 2013, for reviews) have been used during the last decade as probes of the possible origin of Halo stars. The general conclusion is that there are substantial differences between the chemical signatures of the dwarf galaxies and the Galactic halo (hereafter Halo). Moreover, the chemical enrichment of Halo stars is quite complex, and indeed $\alpha$-element abundances display a well-defined dichotomy (Schuster et al. 2012) and a steady decrease when moving from the inner to the outer Halo $\left(R_{\mathrm{G}} \geq 40 \mathrm{kpc}\right.$, Fernández-Alvar et al. 2015). However, recent high-resolution spectra clearly indicate a broad similarity between the metal-poor, and hence presumably the old, stars in the Halo and in several dwarf spheroidals (dSphs, Frebel et al. 2010a,b; Starkenburg et al. 2013; Fabrizio et al. 2015; Frebel \& Norris 2015).

In the interpretation of these chemical patterns, the complexity of dwarf galaxy chemical evolution must also be taken into account, as clearly disclosed by recent high-resolution spectroscopic investigations on Fornax (Lemasle et al. 2014; Hendricks et al. 2014), which confirm and expand on previous medium-resolution studies (Battaglia et al. 2008; Kirby et al. 2011; Letarte et al. 2006). Indeed, the Fornax iron distribution peaks at $[\mathrm{Fe} / \mathrm{H}]=-0.8$, but the tails extend into the very metal-poor $([\mathrm{Fe} / \mathrm{H}] \sim-3)$ and the very metal-rich $([\mathrm{Fe} / \mathrm{H}] \sim 0)$ 
regime. The same conclusion applies to the $\alpha$-elements, for which there is observed a clear enhancement in the metalpoor stars that gradually moves to a depletion in the metalrich ones. The $\alpha$-element distribution also shows a well-defined "knee" at $[\mathrm{Fe} / \mathrm{H}] \simeq-1.9$ that suggests an early onset of $\mathrm{SNe}$ Ia. This extreme chemical complexity goes hand in hand with a complex star formation history (SFH) in Fornax (de Boer et al. 2012a; del Pino et al. 2013). Other dwarf satellites are simpler in their range of ages and metallicities (Martínez-Vázquez et al. 2016a,b), but the comparison between Halo stars and red giant branch (RGB) stars in nearby dwarf galaxies is still hampered by the fact that Halo stellar populations are dominated by old stars, while those in dwarf galaxies suffer the age-metallicity degeneracy and are a mix of old and intermediate-age stars.

That stars of different ages show a clear dichotomy in their chemistry and kinematics is illustrated by the results on Carina by Fabrizio et al. (2016). Based on solid tracers of the old horizontal branch (HB) and intermediate-age red clump (RC) populations in Carina, these authors find a systematic difference in the metallicity distribution and kinematics between these two subpopulations. Specifically, current metallicity estimates based on RGB stars mainly trace the intermediate-age (RC) population, whereas the old stars are systematically more metal-poor. These considerations are consistent with the evidence that the intermediate-age star formation episode in Carina accounts for more than $70 \%$ of its stellar content (Dolphin 2002; Small et al. 2013).

An ideal experiment is one that compares stellar populations with the same age in both the Halo and nearby dwarf galaxies. This opportunity is offered by RR Lyrae stars (RRLs). They are precise distance indicators and solid tracers of evolving stars with initial mass lower than $\sim 0.8 M_{\odot}$, i.e., stars older than 10 Gyr. Even though in extragalactic systems these stars are too faint ( 3.5-4 mag fainter than the tip of the RGB) to spectroscopically measure their individual chemical compositions, they can easily be detected and characterized by their pulsation properties (mean magnitudes, amplitudes, periods) well beyond our LG (e.g. the RRLs observed at $\sim 2 \mathrm{Mpc}$ in the Sculptor group Da Costa et al. 2010). They typically pulsate in the fundamental mode (RR $a b)$, the first overtone (RR $c)$, or both.

In recent years, different photometric sky surveys have provided the pulsation properties of a large number $(\sim 15000)$ of Halo RRLs (e.g. CATALINA survey, Drake et al. 2013). Moreover, they are almost ubiquitous; they have even been detected in galaxies of modest mass: classical dwarfs (e.g. Coppola et al. 2015; Martínez-Vázquez et al. 2016b) and ultra-faint dwarfs (e.g. Garofalo et al. 2013; Boettcher et al. 2013). Our group is systematically investigating variable stars in the LG galaxies using both proprietary and archival data in order to maximize temporal coverage. The global photometry, including the properties of the variable stars are available to the community (e.g. Leo I, Carina, Sculptor ${ }^{1}$ ).

In this investigation we present new results for the RRLs in the Fornax dSph. These results are used to provide an independent and global picture of the early chemical enrichment and the SFH of this interesting galaxy. Fornax is among the most massive galaxies surrounding the MW and one of the few gas-poor stellar systems of the LG hosting (five) GCs. Although Fornax is relatively close $\left(\mu_{0}=20.84 \pm 0.04\right.$ mag using the tip of the RGB, Pietrzyński et al. 2009) and it has been the target of extensive photometric (Coleman \& de Jong 2008;

\footnotetext{
http: //www . cadc-ccda.hia-iha.nrc-cnrc.gc.ca/en/ community/STETSON/homogeneous/
}

de Boer et al. 2012b) and spectroscopic (Letarte et al. 2010; Kirby et al. 2011) investigations, we still lack a complete census of evolved variables. The main previous investigation was performed by Bersier \& Wood (2002) and covered an area of nearly half a square degree, a minor fraction of the body of the galaxy $\left(r_{\mathrm{t}}=71\right.$ arcmin Mateo 1998). Their $V, I$ time series data were collected using 1-1.3 m telescopes in $\sim 2^{\prime \prime}$ seeing, and provided a sample of $396 \mathrm{RR} a b$ and $119 \mathrm{RR} c$ as classified on the basis of their periods. The quality of their photometry was inadequate to determine accurate light curves, and the amplitudes of variation were not provided. A further but still preliminary investigation of cluster and field variables in Fornax was provided by Greco et al. (2005) using the imaging subtraction method. They found more than 700 candidates (see their Table 2) among RRLs, anomalous Cepheids, and dwarf Cepheids (SX Phoenicis). However, a detailed comparison is hampered by the lack of finding charts and astrometric positions of the candidate variables.

In this paper we present a nearly complete sample of RRLs observed in Fornax dSph (Sect. 2). For the first time we show their amplitude vs. period diagram and we discuss its implications for the early chemical enrichment of Fornax dSph (Sect. 3). The period distribution of fundamental mode RRLs in Fornax dSph is used to derive constraints on the contribution of Fornax-like systems to the Halo formation when compared with more massive dwarfs (the Sagittarius and Large Magellanic cloud systems, hereafter Sgr and LMC, respectively; see Sect. 4). Finally, we interpret these findings in the broad context of LG galaxies (Sect. 5).

\section{Photometric data sets and RR Lyrae identification}

The multi-band $(B V I)$ optical photometry employed here is based on both proprietary and archival data sets. The images were collected with telescopes ranging in aperture from $0.9 \mathrm{~m}$ to $8 \mathrm{~m}$, and cover a time interval of nearly $20 \mathrm{yr}$ (1994 December to 2014 October). The median seeing was $1^{\prime \prime} .1$, with an interquartile range of $0^{\prime \prime} .95-1^{\prime \prime} .3$. The data reduction and the calibration is part of the effort of P. B. Stetson to build a database of homogeneous photometry including star clusters and resolved galaxies. A full description of the data, data reduction, and of all the variable stars identified will be addressed in a future paper.

The variable candidates for which we have been able to determine at least a provisional period cover a sky area of $\sim 50$ '. $\times 54^{\prime}$. around the galaxy centre. The number of measurements per band is on average about 130 in $B, 180$ in $V$, and 30 in $I$. Our approach for identifying variable stars has been described in several previous investigations (Stetson et al. 2014a,b). We have identified $\sim 1900$ candidate variables, among them 1426 bona fide RRLs for which we can fit light curves. In this investigation we focus on this unique sample of RRLs since it increases the known sample by a factor of nearly three. Figure 1 shows the location of the current RRL sample in the $V, B-I$ colour-magnitude diagram. The accuracy and the precision of this photometry is highlighted by the smooth transitions among the different populations in the region of the HB $(V \sim 21-22)$. The figure shows the blue HB $(V \sim 22.0-21.5, B-I \sim 0-0.5 \mathrm{mag})$, the RRLs $(V \sim 21.0-21.5$, $B-I \sim 0.5-1.2 \mathrm{mag})$, and the red HB $(V \sim 21.0-21.5$, $B-I \sim 1.2-1.5 \mathrm{mag})$ representing the old stellar population (older than $10 \mathrm{Gyr}$ ), and the RC stars $(V \sim 20.9-21.4, B-I \sim$ $1.5-1.8 \mathrm{mag}$ ) tracing the intermediate-age population. Furthermore, the RGB stars display a well-defined stellar overdensity associated with the RGB bump ( $V \sim 20.9-21.5, B-I \sim 1.7-1.9)$. 


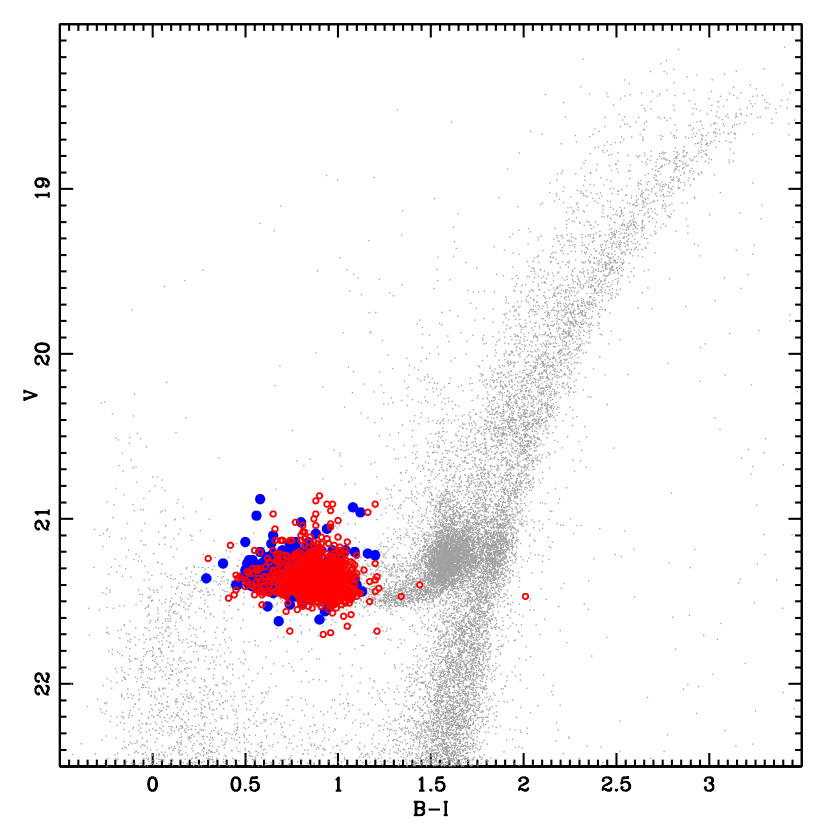

Fig. 1. $V, B-I$ colour-magnitude diagram. Blue dots and red open circles mark RR $c$ and $\mathrm{RR} a b$, respectively.

We note that we have plotted flux-weighted mean magnitudes for the variable stars. The few outliers observed can be explained by the imperfect sampling of the light curve in at least one of the three bands (generally the $I$ band). This also explains why there are several RR $a b$ (red open circles) in the bluer region of the instability strip, i.e. the region where typically only $\mathrm{RR} c$ variables (blue dots) are located.

\section{The Bailey diagram}

To provide a solid classification of the RRLs in our sample we adopted the Bailey diagram (luminosity amplitude vs. period) shown in Fig. 2 (top left panel). The data plotted in this figure show a well-defined transition between $\mathrm{RR} c$ and RR $a b$ in the period range between $\log P=-0.35$ and $\log P=-0.32 \mathrm{~d}$. On the basis of this criterion we end up with $436 \mathrm{RR} c$ and 990 $\mathrm{RR} a b$ variables. It is worth noting that Fornax lacks high amplitude short period RR $a b$ variables (HASPs), i.e. RR $a b$ with periods shorter than $P \lesssim 0.48 \mathrm{~d}^{2}$ (teal coloured region in Fig. 2, left panel; see also Fiorentino et al. 2015). We note that the lack of HASPs is also evident among the RRLs hosted in the five Fornax GCs (green and red circles; Mackey \& Gilmore 2003; Greco et al. 2007, respectively).

In this context it is worth mentioning that across the transition region between RRc and RR $a b$ variables are also found the mixed-mode ( $R R d$ ) variables that pulsate simultaneously in the first overtone (typically the main mode) and in the fundamental mode. They display a well-defined amplitude modulation, but this is typically of the order of a few tenths of a magnitude (Smolec \& Bakowska 2016). In this region are also found Blazhko RRLs, i.e. RRLs that display amplitude and/or phase modulations on a time scale ranging from tens of days to years.

\footnotetext{
2 The boundary of the HASP at $P \lesssim 0.48 \mathrm{~d}$ (Fiorentino et al. 2015) was arbitrary and based on preliminary evidence that no small dSph of the MW was known to host RRLs of this kind. Importantly, the updated catalogues for Sculptor (Martínez-Vázquez et al. 2015) and Fornax (this work) confirm that choice. The precise value of this limit has no impact on the present discussion.
}

The amplitude modulation ranges from a few tenths to more than half a magnitude (Piersimoni et al. 2002; Braga et al. 2016). The pulsation characteristics available in the literature do not allow us to assess whether the Fornax cluster RRLs located below the HASP region (green circles in Fig. 2, left panel) are RRd or Blazhko RRLs. Therefore, the periods and amplitudes for these stars, which appear to have in principle the right periods to be classified as HASPs, may not be definitive since the data presented in Mackey \& Gilmore (2003) are undersampled, as discussed in their Sect. 3.4. However, four out of the five Fornax GCs are metal-poor and thus not expected to produce HASP RRLs (see below). These are the reasons why we do not discuss the GC sample further.

Circumstantial empirical and theoretical evidence indicates that the lack of HASPs can be explained by a lack of old stellar populations more metal-rich than $[\mathrm{Fe} / \mathrm{H}] \sim-1.5$ (as shown in Fiorentino et al. 2015). This finding may appear to be at odds with current spectroscopic measurements (Battaglia et al. 2008; Kirby et al. 2011; Letarte et al. 2006, 2010; Lemasle et al. 2014; Hendricks et al. 2014) showing that Fornax RGB stars peak at $[\mathrm{Fe} / \mathrm{H}] \sim-0.9$ and range from -3.0 to -0.4 dex. Clearly, more metal-rich populations are present in Fornax, but they may just be too young to produce RRLs. A number of studies (e.g., Fabrizio et al. 2015, 2016; Martínez-Vázquez et al. 2016b) have demonstrated that purely old stellar populations do not necessarily share the mean chemical abundance of RGB stars. In agreement, the globular clusters support the inference that the old populations in Fornax are significantly more metal poor than the average: the mean metallicity of four out of the five clusters is $[\mathrm{Fe} / \mathrm{H}]=-2$ or less (Letarte et al. 2006), and only GC 4 has a mean metallicity as high as $[\mathrm{Fe} / \mathrm{H}] \sim-1.40 \pm 0.06$ (Larsen et al. 2012, using integrated-light, high-dispersion spectra), though it is also believed to be $3 \mathrm{Gyr}$ younger than the other four (Buonanno et al. 1999). We note here that Letarte et al. (2006) found a bias in the estimated metallicity to higher values (amounting to $\sim+0.3$ to $+0.5 \mathrm{dex}$ ) when using integrated-light spectra instead of individual stellar measurements.

To further test the hypothesis that Fornax RRLs are metal poor $([\mathrm{Fe} / \mathrm{H}] \lesssim-1.5)$, Fig. 2 (right panels) shows the comparison between the current sample of RRLs and evolutionary predictions for helium-burning structures available in the BaSTI database. To compare theory and observations we adopted a true distance modulus of $\mu_{0}=20.84 \pm 0.04 \mathrm{mag}$, a reddening of 0.03 mag (Pietrzyński et al. 2009), and the Cardelli et al. (1989) reddening law. The bottom panel of this figure shows the comparison between the theoretical zero age horizontal branch (ZAHB, solid black line) for two different chemical compositions $[\mathrm{Fe} / \mathrm{H}]=-1.5$ (bottom panel) and -2.2 (top panel) and for both a scaled-solar and an alpha-enhanced chemical mixture (Pietrinferni et al. 2014). The dashed and dotted lines respectively represent the loci of $90 \%$ and $100 \%$ helium exhaustion in the core. The time for the last $10 \%$ of helium exhaustion is only a small percentage compared to the total lifetime spent close to the ZAHB (98\%). The models suggest that RRLs in Fornax can hardly be explained as an old stellar population with a narrow and quite metal-rich $([\mathrm{Fe} / \mathrm{H}] \gtrsim-1.5 \mathrm{dex})$ metallicity distribution. A metallicity distribution ranging from at least -1.5 dex to less than -2.2 dex seems more compatible with the wide HB distribution of RRLs. We note that the adopted evolutionary scenario does not affect our conclusions; indeed, alpha-enhanced models for $[\mathrm{Fe} / \mathrm{H}] \sim-1.5$ predict a ZAHB only slightly fainter $(\Delta V \sim+0.02 \mathrm{mag}$, dark green lines in Fig. 2) than scaled-solar ones. 

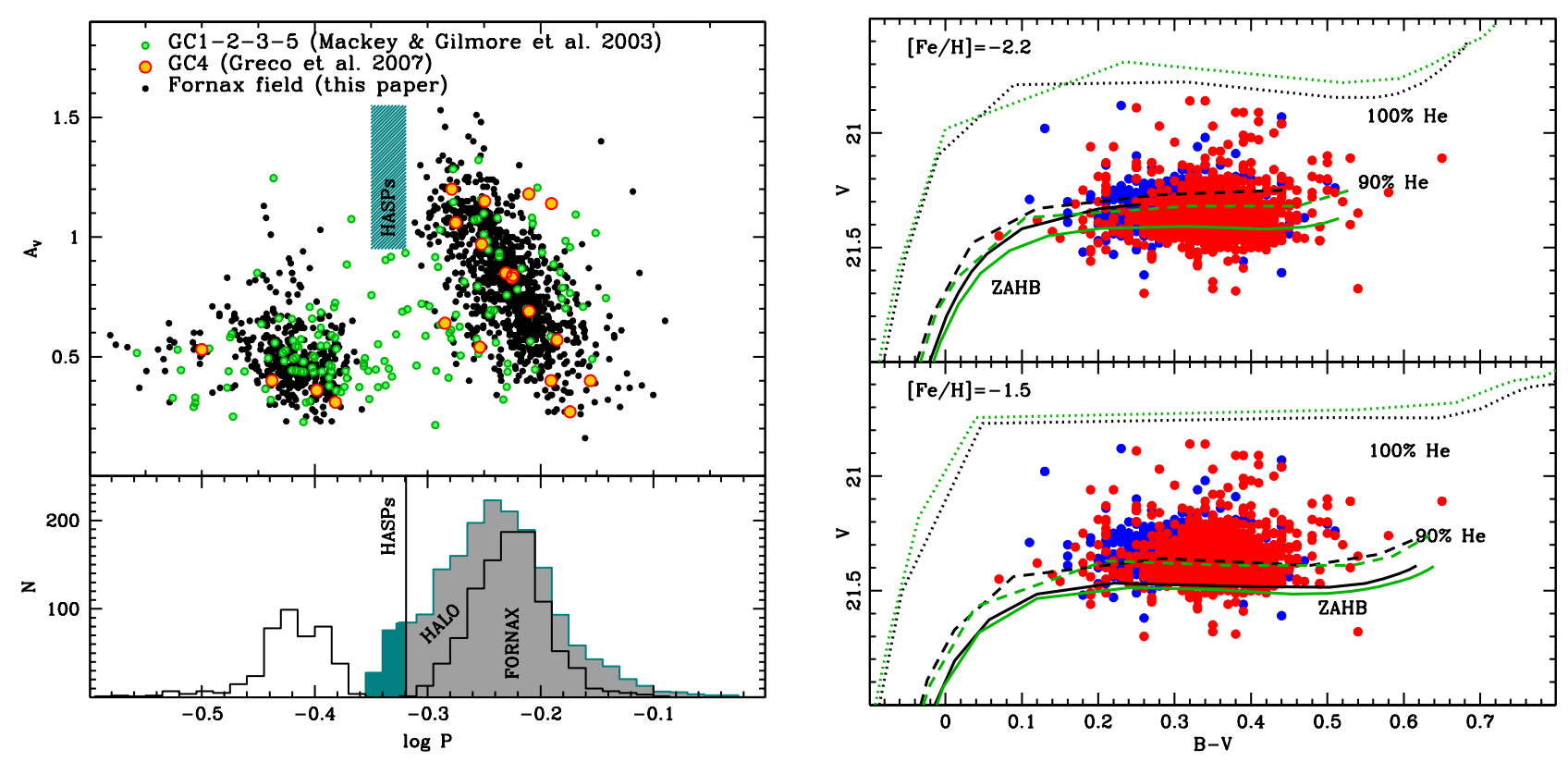

Fig. 2. Left panels: period- $V$ band amplitude (Bailey) diagram (top) and the period distribution (bottom) of the 1426 Fornax RRLs (black dots). RRLs in the Fornax globular clusters are also shown. The teal coloured region indicates the HASPs' location. The grey/teal histogram is the period distribution of $\sim 15000$ Halo RRLs. Right panels: zoom on the horizontal branch of the Fornax dSph is shown compared with theoretical predictions for scaled-solar (black lines) and alpha-enhanced (dark green lines) chemical compositions. The loci of the zero age horizontal branch (solid lines) of the stars that have burned $90 \%$ (dashed) and 100\% (dotted) of their central helium are shown for two selected metal abundances.

Our conclusion is supported by an independent analysis by de Boer et al. (2012b). They computed a detailed SFH and an age-metallicity relation (AMR) combining deep- and widefield photometry with medium-resolution spectroscopy. They find that Fornax is dominated by intermediate-age stars, but also hosts an old population ( $>10 \mathrm{Gyr})$. Furthermore, their AMR indicates that the old population has a metallicity $[\mathrm{Fe} / \mathrm{H}] \lesssim$ $-1.5 \mathrm{dex}$, thus explaining the lack of HASPs in spite of the very large RRL sample.

\section{Building up the Halo with Fornax-like dSph}

In Fiorentino et al. (2015) we showed that HASPs are observed in the Halo (see teal region in Fig. 2), while they are typically absent in dwarf satellites of the MW, with the exception of the most massive ones such as Sgr and the Magellanic clouds. Thus, despite Fornax's large total mass, its huge RRL sample shows a behaviour similar to the majority of low-mass dwarfs. Owing to their very high amplitudes and to the good sampling of our data set, the HASP deficiency cannot be an observational bias and has strong implications for Halo formation. On the basis of the observational evidence that low-mass dwarfs do not contain HASP RRLs, in Fiorentino et al. (2015) we argued that a major role for small dwarfs, as we see them today, can be excluded in building up the Halo. This conclusion was based on a large sample of RRLs for the Halo ( 15000 stars) and for small ( 1300 stars) and large (LMC, SMC, and Sgr) dwarfs. A similar conclusion was achieved by Zinn et al. (2014), but based on a smaller Halo field sample (Smooth Halo, 170 objects) and Fornax dwarf sample ( 396 objects; Bersier \& Wood 2002; Greco et al. 2005).

In Fig. 2 we show the period-amplitude diagram and the period distribution of the RRLs in Fornax (black solid line). For comparison we have plotted the Halo distribution for RR $a b$ stars (grey region) arbitrarily rescaled. The Fornax period distribution is very different from that of the Halo and resembles that found using the data on small mass dwarfs collected by Stetson et al. (2014b). The latter is indeed well described by a Gaussian function peaked around $\sim 0.59 \mathrm{~d}$ and with a small $\sigma(0.05)$. A Kolmogorov-Smirnoff test excludes the possibility that the two period distributions (bottom left panel of Fig. 2) are drawn from the same parent population.

In order to quantify the role of Fornax-like systems in Halo formation we have adopted the following approach. The main difference between the Halo and the Fornax period distribution is seen in their short-period tail. Therefore, we decided to reproduce the Halo period distribution using a linear combination of Fornax-like systems plus massive galaxies that do host HASPs, i.e. the LMC and Sgr (Fiorentino et al. 2015). For the sake of simplicity we approximated all the distributions by analytical Gaussian functions. To compare samples of similar size we randomly extracted $\sim 2000$ objects from the full Halo sample (grey histograms in Fig. 3). This extraction was repeated 200 times. We used the same procedure for the LMC sample. For each Halo realization we computed the linear combination that best reproduces the Halo Gaussian function, see Fig. 3 (magenta lines). In each panel of this figure we also show the individual components that form the linear combination. Interestingly enough, when combined with Sgr (teal green lines, bottom left panel) Fornax can contribute a fraction that goes from 0 to $20 \%$ of the Halo, whereas this fraction decreases to $0-8 \%$ when combined with LMC (cyan lines, top left panel). This suggests that Fornax-like systems, unlike other massive dwarfs, have likely contributed minimally to the Halo. In this sense Fornax seems to be a transitional system between small and massive dwarfs.

\section{HASPs and the early host galaxy evolution}

To further investigate the connection between the chemical enrichment history in dwarf galaxies and their HASP content, we extend our discussion to the LG. Figure 4 shows the classical 


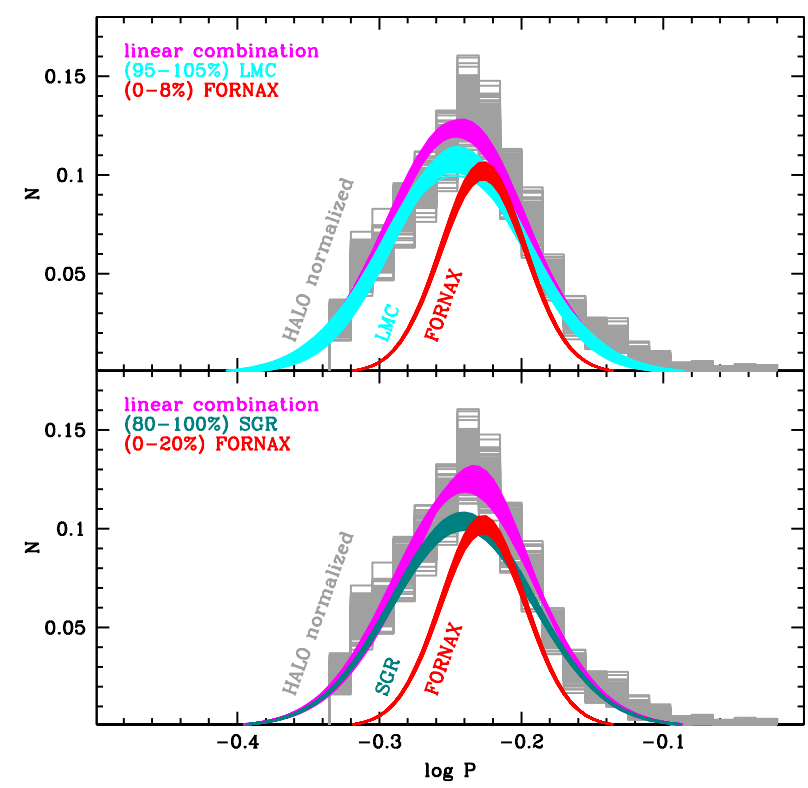

Fig. 3. Halo period distributions for the 200 realizations of randomly extracted subsamples (grey histograms). The magenta lines show the linear combinations of Fornax (red) period distribution with stellar systems that host HASPs, i.e. LMC (cyan) and Sgr (teal green). The individual contributions to the Halo for each stellar system used for the linear combinations are also given in each panel.

mean metallicity-luminosity relation (data from McConnachie 2012) for LG dwarf galaxies. Only stellar systems in which at least one RRL is found have been included. Filled circles represent systems hosting at least one HASP, while red and blue symbols represent fast and slow evolvers in the nomenclature of Gallart et al. (2015): the fast galaxies formed the vast majority of their mass in stars at early epochs $(>10 \mathrm{Gyr})$, while the slow galaxies are mainly dominated by a mix of intermediate-age and young stellar populations. This means that the old population in the latter systems represents only a minor fraction of the entire baryonic mass budget.

Data plotted in this figure indicate that the current sample can be split into three different groups:

- Bright systems - This group includes stellar systems brighter than $M_{V} \sim-13.5 \mathrm{mag}$, which all host HASPs. These galaxies surround both the MW (SMC, Soszyński et al. 2010; LMC, Soszyński et al. 2009; Sgr dSph, Soszyński et al. 2014) and M31 (NGC 147, NGC 185, Monelli et al., in prep.; M32, Fiorentino et al. 2010, 2012). With the exception of the Magellanic clouds and NGC 147, the other galaxies are dominated by old stellar populations.

- Faint systems - This group includes stellar systems fainter than $M_{V} \sim-11 \mathrm{mag}$; none of these host HASPs. This is the largest subsample, dominated by gas-poor (dSph, ultra faint) systems made of mostly old stellar populations. A few galaxies hosting a significant intermediateage population are also present, i.e. Carina (Monelli et al. 2003), Leo T dSph/dIrr (Clementini et al. 2012), DDO210 $\mathrm{dSph} / \mathrm{dIrr}$ (Cole et al. 2014).

- Intermediate luminosity systems - This group includes stellar systems with absolute magnitude ranging from $M_{V} \sim-11$ to $-13.5 \mathrm{mag}$. These may or may not host HASPs, and we argue that the presence of HASPs is related to their individual early SFH (see below).
This figure indicates that, among the more massive stellar systems, both slow and fast evolvers have had an early chemical enrichment that was rapid enough to produce such old ( $\approx 10 \mathrm{Gyr}$ ), metal-rich $([\mathrm{Fe} / \mathrm{H}] \gtrsim-1.5)$ stellar populations that we observe nowadays as HASPs. In contrast, the less massive galaxies had an early chemical enrichment insufficient to reach the necessary metallicity to produce HASPs. In the intermediate luminosity region there is also a mix of slow and fast galaxies. However, all the slow evolvers in this region completely lack HASPs (Leo I, Stetson et al. 2014a; Leo A, Bernard et al. 2013; Fornax, this work), while the fast do host a small fraction of them $(\$ 3 \%$; Sculptor, Martínez-Vázquez et al. 2016b; Cetus, Bernard et al. 2009; Andromeda I and Andromeda II, Martínez-Vázquez et al., in prep.; Andromeda VI, Pritzl et al. 2002; Andromeda VII, Monelli et al., in prep.). Therefore, in the transition region the total current baryonic mass $\left(\sim 2-20 \times 10^{6}\right.$, McConnachie 2012) is not the only parameter driving the early chemical enrichment and, in turn, the presence or lack of HASPs. The total luminosities of Fornax and Sculptor seem to be the lower limits for the presence of HASPs among slow and fast galaxies, respectively. This suggests that the presence/lack of HASPs in this luminosity range, with HASP present in fast galaxies but not in slow ones, depends strongly on the details of the early SFH, and further strengthens the hypothesis that slow and fast galaxies were already different $\sim 10$ Gyr ago (Gallart et al. 2015). It also establishes a means to get insight into the early SFH of dwarf galaxies based on the presence or absence of HASPs among their RRL population.

Among the four cluster-bearing dwarf galaxies, the Sgr and Fornax dSphs have the same current luminosity (baryonic mass, see Fig. 3) and similar numbers of globulars (Mackey \& Gilmore 2004). However, their mean metallicities and their evolution histories are quite different. The number of RRab in Sgr is almost a factor of two greater than in Fornax ( 1600 vs. 1000) and there are clear differences in their RR $a b$ period distributions and in the HASP occurrence. The role played by Sgr and Fornax in building up the Halo has already been discussed by Zinn et al. (2014), using more limited samples of field RRLs, and by Mackey \& Gilmore (2004) using their cluster RRLs. Our findings support their results, further stressing the major contribution of massive stellar systems like Sgr and/or the LMC when compared with Fornax-like dSph galaxies ( $~ 80-100 \%$ vs. $0-20 \%$ ) in very good agreement with current cosmological simulations (Cooper et al. 2010; Tissera et al. 2014). In passing, we note that this is also consistent with the hypothesis put forward by Gallart et al. (2015) that slow dwarfs such as Fornax may have formed in low-density environments, away from the main cosmic density fluctuations that gave rise to the MW or M31, which also implies a later accretion into the main galaxies.

\section{Final remarks}

The use of pulsation properties of RRLs (amplitudes, periods) as diagnostics of the metallicity distribution of old stellar populations dates back to more than one century ago. The results presented in this paper indicate that they can also be adopted to provide independent constraints for galaxy formation models, and in particular, for the role played by mergers in building up the halo of massive galaxies (Bullock \& Johnston 2005; Zolotov et al. 2009).

This investigation paves the way to the use of RRLs for tracing the early star formation and chemical enrichment histories in Local Group galaxies and in nearby galaxy clusters. This opportunity becomes even more compelling if we account 


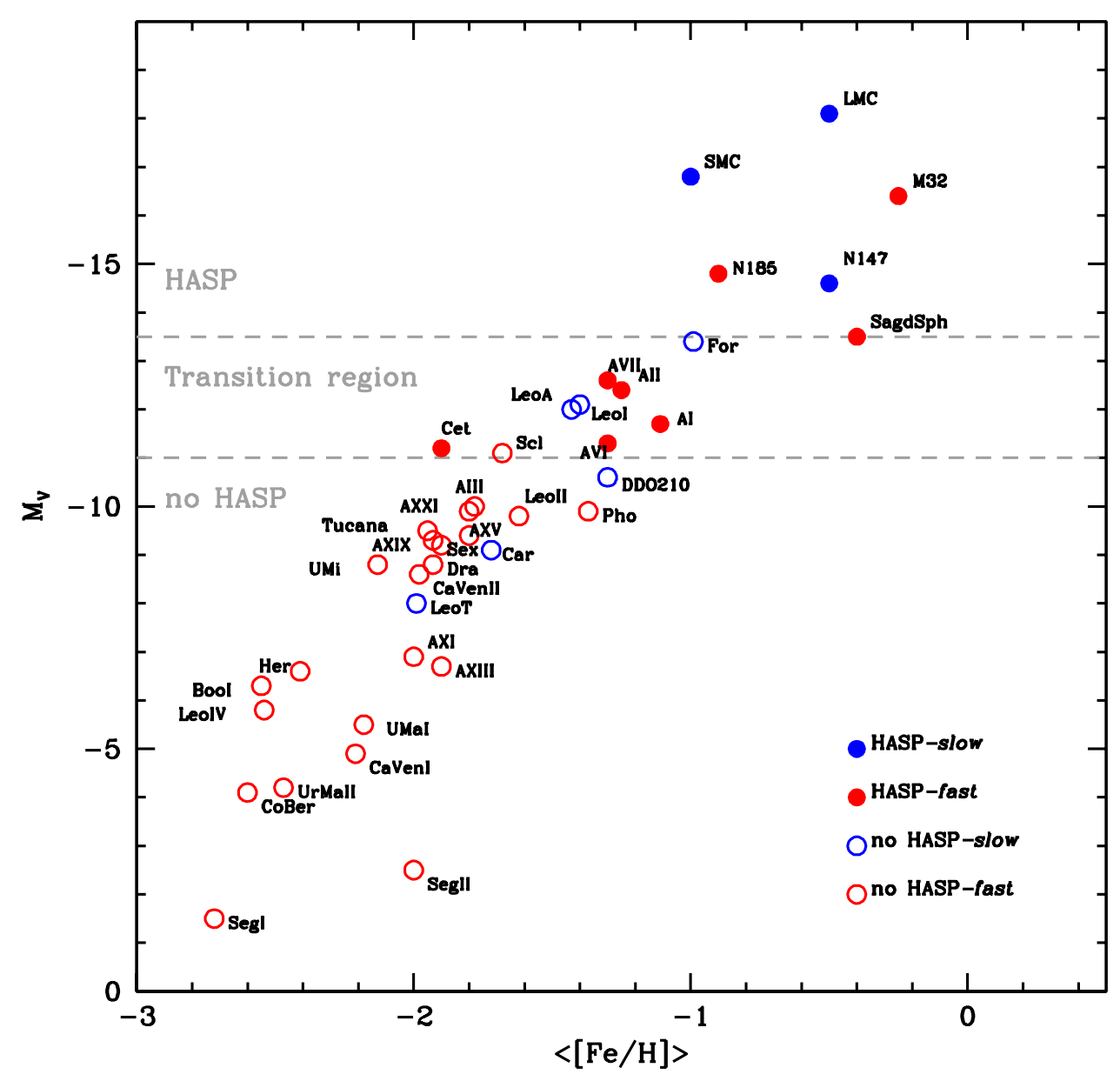

Fig. 4. Luminosity-metallicity distribution (McConnachie 2012) for all the dwarf galaxies of the LG for which a short-period variability study is available. Filled and empty circles mark either the presence or the lack of HASPs; slow and fast galaxies are colour-coded red and blue, respectively.

for the homogeneous multi-band $(u, g, r, i, z, y)$ time series data that will be collected by the Large Synoptic Survey Telescope (LSST; Ivezic et al. 2010) and for the deep limiting magnitudes of individual exposures. Moreover, in the near future space-based (James Web Space Telescope) and ground-based Extremely Large Telescopes will offer the opportunity to extend photometric and spectroscopic investigations of primary distance indicators to Local Volume galaxies.

Acknowledgements. This research has been supported by the Spanish Ministry of Economy and Competitiveness (MINECO) under the grant AYA2014-56795-P. E.J.B. acknowledges support from the CNES postdoctoral fellowship program. G.F. and D.M. have been supported by the Futuro in Ricerca 2013 (grant RBFR13J716).

\section{References}

Battaglia, G., Helmi, A., Tolstoy, E., et al. 2008, ApJ, 681, L13 Bernard, E. J., Monelli, M., Gallart, C., et al. 2009, ApJ, 699, 1742 Bernard, E. J., Monelli, M., Gallart, C., et al. 2013, MNRAS, 432, 3047 Bersier, D., \& Wood, P. R. 2002, AJ, 123, 840 Boettcher, E., Willman, B., Fadely, R., et al. 2013, AJ, 146, 94 Braga, V. F., Stetson, P. B., Bono, G., et al. 2016, AJ, 152, 170 Bullock, J. S., \& Johnston, K. V. 2005, ApJ, 635, 931 Buonanno, R., Corsi, C. E., Castellani, M., et al. 1999, AJ, 118, 1671 Cardelli, J., Clayton, G., \& Mathis, J. 1989, ApJ, 345, 245 Clementini, G., Cignoni, M., Contreras Ramos, R., et al. 2012, ApJ, 756, 108 Cole, A. A., Weisz, D. R., Dolphin, A. E., et al. 2014, ApJ, 795, 54
Coleman, M. G., \& de Jong, J. T. A. 2008, ApJ, 685, 933 Cooper, A. P., Cole, S., Frenk, C. S., et al. 2010, MNRAS, 406, 744 Coppola, G., Marconi, M., Stetson, P. B., et al. 2015, ApJ, 814, 71 Da Costa, G. S., Rejkuba, M., Jerjen, H., \& Grebel, E. K. 2010, ApJ, 708, L121 de Boer, T. J. L., Tolstoy, E., Hill, V., et al. 2012a, A\&A, 539, A103 de Boer, T. J. L., Tolstoy, E., Hill, V., et al. 2012b, A\&A, 544, A73 del Pino, A., Hidalgo, S. L., Aparicio, A., et al. 2013, MNRAS, 433, 1505 Dolphin, A. E. 2002, MNRAS, 332, 91

Drake, A. J., Catelan, M., Djorgovski, S. G., et al. 2013, ApJ, 763, 32 Fabrizio, M., Nonino, M., Bono, G., et al. 2015, A\&A, 580, A18

Fabrizio, M., Bono, G., Nonino, M., et al. 2016, ApJ, 830, 126

Feltzing, S., \& Chiba, M. 2013, New Astron. Rev., 57, 80

Fernández-Alvar, E., Allende Prieto, C., Schlesinger, K. J., et al. 2015, A\&A, 577, A 81

Fiorentino, G., Contreras Ramos, R., Clementini, G., et al. 2010, ApJ, 711, 808

Fiorentino, G., Contreras Ramos, R., Tolstoy, E., Clementini, G., \& Saha, A. 2012, A\&A, 539, A138

Fiorentino, G., Bono, G., Monelli, M., et al. 2015, ApJ, 798, L12

Frebel, A., \& Norris, J. E. 2015, ARA\&A, 53, 631

Frebel, A., Kirby, E. N., \& Simon, J. D. 2010a, Nature, 464, 72

Frebel, A., Simon, J. D., Geha, M., \& Willman, B. 2010b, ApJ, 708, 560

Gallart, C., Monelli, M., Mayer, L., et al. 2015, ApJ, 811, L18

Garofalo, A., Cusano, F., Clementini, G., et al. 2013, ApJ, 767, 62

Greco, C., Clementini, G., Held, E. V., et al. 2005, ArXiv e-prints [astro-ph/0507244]

Greco, C., Clementini, G., Catelan, M., et al. 2007, ApJ, 670, 332

Hendricks, B., Koch, A., Walker, M., et al. 2014, A\&A, 572, A82

Ivezic, Z., Axelrod, T., Burke, D., et al. 2010, BAAS, 42, 217

Kirby, E. N., Lanfranchi, G. A., Simon, J. D., Cohen, J. G., \& Guhathakurta, P. 2011, ApJ, 727, 78

Larsen, S. S., Strader, J., \& Brodie, J. P. 2012, A\&A, 544, L14 
Lemasle, B., de Boer, T. J. L., Hill, V., et al. 2014, A\&A, 572, A88

Letarte, B., Hill, V., Jablonka, P., et al. 2006, A\&A, 453, 547

Letarte, B., Hill, V., Tolstoy, E., et al. 2010, A\&A, 523, A17

Mackey, A. D., \& Gilmore, G. F. 2003, MNRAS, 345, 747

Mackey, A. D., \& Gilmore, G. F. 2004, MNRAS, 355, 504

Martínez-Vázquez, C. E., Monelli, M., Bono, G., et al. 2015, MNRAS, 454, 1509

Martínez-Vázquez, C. E., Monelli, M., Gallart, C., et al. 2016a, MNRAS, 461,

L41

Martínez-Vázquez, C. E., Stetson, P. B., Monelli, M., et al. 2016b, MNRAS, 462 4349

Mateo, M. L. 1998, ARA\&A, 36, 435

McConnachie, A. W. 2012, AJ, 144, 4

Monelli, M., Pulone, L., Corsi, C. E., et al. 2003, AJ, 126, 218

Piersimoni, A. M., Bono, G., \& Ripepi, V. 2002, AJ, 124, 1528

Pietrinferni, A., Molinaro, M., Cassisi, S., et al. 2014, Astron. Comp., 7, 95

Pietrzyński, G., Górski, M., Gieren, W., et al. 2009, AJ, 138, 459

Pritzl, B. J., Armandroff, T. E., Jacoby, G. H., \& Da Costa, G. S. 2002, AJ, 124, 1464
Schuster, W. J., Moreno, E., Nissen, P. E., \& Pichardo, B. 2012, A\&A, 538, A21 Searle, L., \& Zinn, R. 1978, ApJ, 225, 357

Small, E. E., Bersier, D., \& Salaris, M. 2013, MNRAS, 428, 763

Smolec, R., \& Bakowska, K. 2016, Commmunications of the Konkoly Observatory Hungary, 105, 215

Soszyński, I., Udalski, A., Szymański, M. K., et al. 2009, Acta Astron., 59, 1

Soszyński, I., Udalski, A., Szymañski, M. K., et al. 2010, Acta Astron., 60, 165

Soszyński, I., Udalski, A., Szymanski, M. K., et al. 2014, Acta Astron., 64, 177

Springel, V., Frenk, C. S., \& White, S. D. M. 2006, Nature, 440, 1137

Starkenburg, E., Hill, V., Tolstoy, E., et al. 2013, A\&A, 549, A88

Stetson, P. B., Braga, V. F., Dall'Ora, M., et al. 2014a, PASP, 126, 521

Stetson, P. B., Fiorentino, G., Bono, G., et al. 2014b, PASP, 126, 616

Tissera, P. B., Beers, T. C., Carollo, D., \& Scannapieco, C. 2014, MNRAS, 439, 3128

Tolstoy, E., Hill, V., \& Tosi, M. 2009, ARA\&A, 47, 371

Venn, K. A., Irwin, M., Shetrone, M. D., et al. 2004, AJ, 128, 1177

Zinn, R., Horowitz, B., Vivas, A. K., et al. 2014, ApJ, 781, 22

Zolotov, A., Willman, B., Brooks, A. M., et al. 2009, ApJ, 702, 1058 